\title{
Ueber die Veränderlichkeit des Sterns BD. $-3^{\circ} .5159$.
}

Zur Ergänzung der Notiz in A. N. 3567 p. 268 theilt Prof. $A$. Abetti in einem Schreiben vom 14. Juni 1899 die folgenden Grössenschätzungen des Sterns BD. - 3:5159 mit, welche dessen Veränderlichkeit als unzweifelhaft erscheinen lassen :

1 898 Luglio $23,24,26$ Splendidissimo e $25 \mathrm{Neb}$ bioso. La $-3.5159 \quad 9^{\circ} \cdot 3=v$ si stimò di $1 \mathrm{~m}_{5}^{\mathrm{m}}$ perchè più piccola di $-3 \cdot 55^{\circ} 99^{\mathrm{m}} \cdot 5=y$, più piccola di tutte quelle della carta 62 di Schönfeld, ed ancora, più piccola di quelle da me ritenute di $\mathrm{I} \mathrm{I}^{\mathrm{m}}$. Si sospettano accanto alla $v$ due stelline di ${ }^{2} 3^{\mathrm{m}}$ (ultime visibili nell' Amici) una più australe l'altra più boreale. - Agosto so Splendido. La $v$ è più piccola di $-3{ }^{\circ} 5{ }_{5}^{6} \quad 9^{\mathrm{m}} \mathrm{r}=m$ e di $y$, quindi di $9^{\mathrm{m}} \cdot 5$ in 9.8 . - Agosto 11 Splendido, $v=10^{\mathrm{m}}$. Si rivedono le due stelle piccolissime accanto. - Agosto I2, I3,
I 4 Splendido, $v=9 \cdot 5$. - Agosto I 7, I 8 Splendido, $v$ quasi eguale ad $y$ a meno, quindi $\mathrm{di} 9.5$ in $\mathrm{ro}^{\mathrm{m}}$. Agosto $2 \mathrm{r}$. Aria poco trasparente, $z$ meno di $y$. - Sett. 6 . Bello, $v$ maggiore di $y$ ed $m$, osservazione sicurissima. Sett. $7,8,9$. Bello. La $v$ splende come nel 6 più di $m$, ed è più gialla. - Sett. ıo. Non perfetto sereno. $y$ gialla rossastra manifestamente più grande di $m$. -1898 Sett. I 7 Splendidissimo, $v$ molto più grande di $y$ e di $m$, quasi come $-3.51628 .7 \mathrm{Sj}$.

In einem späteren Briefe vom 29. Juli 1899 schreibt noch Prof. A. Abetti:

- La variabilita della -3.5159 è ormai un fatto certo; dall' 8 all' I I Luglio 1899 cioè prima del Plenilunio Antoniazzi non la vide, io la vidi a fatica, e Cerulli la vide di $13^{\mathrm{m}}$ con una compagna nord seguente più piccola. «

\section{Meridian Circle Observations of Comparison Stars and of Planet (17) Thetis.}

The first two of these stars have been used here as comparison stars for the planet (17) Thetis; the last one for the planet (202) Chryseis. All three are Weisse-Bessel stars and their numbers are from that catalogue.

\begin{tabular}{|c|c|c|c|c|c|}
\hline $\begin{array}{l}\text { No. } \\
15^{\mathrm{h}} \cdot 360\end{array}$ & $\begin{array}{l}\text { Mag. } \\
71 / 2\end{array}$ & $\begin{array}{c}\alpha 1899.0 \\
I 5^{\mathrm{h}} 22^{\mathrm{m}} 12^{\mathrm{s}} \cdot 30\end{array}$ & $\begin{array}{c}1899.0 \\
-8^{\circ} 35^{\circ} 4^{\prime \prime} .9\end{array}$ & $\begin{array}{c}\text { No. of obs. } \\
3\end{array}$ & $\begin{array}{c}\text { Epoch } \\
1899.41\end{array}$ \\
\hline 15.621 & $7 \%$ & 1535 & $\begin{array}{lll}-8 & 4 & 44.9\end{array}$ & 3 & 1899.41 \\
\hline 16.364 & $8^{1 / 4}$ & I 62 I 59.07 & -95454.1 & 2 & 1899.48 \\
\hline
\end{tabular}

The first star is also in the Cordoba General Catalogue and in the Munich Catalogue of 1890 .

By comparison with the Weisse-Bessel position the second star (No. $15^{\mathrm{h}} .62 \mathrm{r}$ ) shows a proper motion of $-0^{\mathrm{s}} .0132$ in $\alpha$ and -0.114 in $d$. The other two show no proper motion.

While observing the first two of these stars the following positions were obtained for

(17) Thetis.

\begin{tabular}{|c|c|c|c|}
\hline 1899 & Mt. Hamilton M. T. & $\alpha$ & 8 \\
\hline$y \quad 27$ & $10^{\mathrm{h}} 5^{8^{\mathrm{m}}} 6^{\mathrm{s}} .1 \mathrm{I}$ & $15^{\mathrm{h}} 20^{\mathrm{m}} 3^{1 \mathrm{~s}} \cdot 90$ & $-8^{\circ} 33^{\prime} 44^{\prime \prime} 8$ \\
\hline 2 & 10 $29 \quad 52.86$ & 53.37 & $\begin{array}{lll}-8 & 34 & 25.9\end{array}$ \\
\hline
\end{tabular}

Lick Observatory, University of California, 1899 July 1 .

R. T. Crazeford.

\section{Literarische Anzeigen.}

F. Bolte. Neues Handbuch der Schifffahrtskunde. Mit einer Vorrede von Prof. Dr. Neumayer. Hamburg I 899.

Das Handbuch zerfällt in fünf Theile: 1) die Schifffahrt nach der Karte, 2) die Schiffahrt nach der Besteckrechnung, 3) die Schifffahrt nach astronomischen Beobachtungen, 4) Grundzïge der maritimen Meteorologie und der Oceanographie, 5) die nautischen Instrumente und ihr Gebrauch. Mit besonderer Ausfuhrlichkeit ist der dritte Theil behandelt, der auch für Fachastronomen von Interesse sein dürfte.

F. Bolte. Nautische Tafelsammlung. Nebst vier magnetischen Karten, entworfen von Professor Dr. Neumayer. Hamburg 1899 .

Die Tafeln sind im engen Anschluss an das Handbuch des Verfassers mit besonderer Berücksichtigung der Verwendbarkeit an Bord zusammengestellt worden.

Planet (163) Erigone scheint von der Ephemeride (Veröff. R. I. Nr.9) mehr als $\mathrm{I}^{\mathrm{m}}$ abzuweichen. $\mathcal{F}$. Palisa.

Planet (265) Anna. Corr. der Ephemeride (A. N. 3577 ): Aug. r $4+17^{\mathrm{s}}+3^{\prime} 8$. F. Palisa.

Planet (343) 0stara. Beiläufige Corr. der Ephemeride (Veröff. R. I. Nr. Io): Aug. 15 +20 5 +2'. F. Palisa.

Inhalt zu Nr. 3589. W. Villiger. Beobachtungen von Cometen und Planeten. 225. - A. Abetti. Cometa 1899 I. 235. - A. Abetti. Osservazioni del pianeta (85) ]o $=[\mathbf{I} 899 \mathrm{EN}]$ 9.96. 237. - Ueber die Veränderlichkeit des Sterns BD. - 3.5159. 239. R. T. Crazuford. Meridian Circle Observations of Comparison Stars and of Planet (17) Thetis. 239. - Literarische Anzeigen. 239. - F. Palisa. Correctionen von Planeten-Ephemeriden. 239. 